\title{
A significant role for tumor necrosis factor in Nlrp3 inflammasomeopathies
}

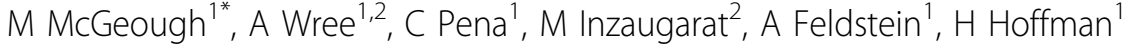 \\ From 8th International Congress of Familial Mediterranean Fever and Systemic Autoinflammatory Diseases \\ Dresden, Germany. 30 September - 3 October 2015
}

\section{Introduction}

The NLRP3 inflammasome is a protein complex responsible for caspase-1 dependent maturation of the pro-inflammatory cytokines IL-1 $\beta$ and IL-18. Gain of function missense mutations in NLRP3 cause the disease spectrum known as cryopyrin-associated periodic syndromes (CAPS).

\section{Objective}

To elucidate systemic autoinflammatory disease mechanisms involved NLRP3 inflammasomeopathies other than IL-1 $\beta$ and IL-18.

\section{Materials and methods}

Knock-in Nlrp3 $3^{L 351 P /+} / \mathrm{CreL} / \mathrm{Il} 1 \mathrm{~b}-/-/ \mathrm{Il18}$-/-mice (FCAS

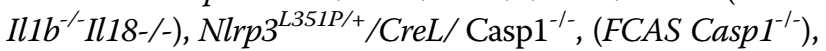
$\mathrm{Nlrp} 3^{\mathrm{A35OV/+}} / \mathrm{CreL}$ (MWS) and Nlrp3 $3^{\mathrm{A35OV/+}} / \mathrm{CreL} / \mathrm{Tnf}^{\prime-}$ (MWS $\operatorname{Tnf}^{\prime-}$ ) were generated in which Cre-mediated expression is limited to the myeloid cell lineage.

\section{Results}

Nearly all FCAS Il1 b- Il18-/-mice survived and grew normally until adulthood however, investigation of mice at $>6$ months of age showed marked splenomegaly and elevated numbers of white blood cells as compared to FCAS Casp $1^{-/-}$ mice and non-mutant $\mathrm{Ill}^{-/}$Ill18-/-littermates, suggesting a caspase- 1 dependent phenotype independent of IL-1 $\beta$ and IL-18. To examine other potential inflammatory mediators, non-lethal in vivo LPS (5 ug/g) stimulation of FCAS $\mathrm{Illb}^{-/}$ $\mathrm{Il} 18^{-/-}$mice revealed significantly elevated levels of serum TNF at both 2 and 6 hours post induction when compared to FCAS Casp1-- mice and non-mutant Ill $\mathrm{b}^{-/}$Ill8-/-controls. To further investigate the role of TNF in Nlrp3 inflammasomeopathies, MWS mice (which die within two weeks of birth) were bred on a TNF knockout background. MWS

${ }^{1}$ University of California San Diego, Pediatrics, La Jolla, USA

Full list of author information is available at the end of the article
$\mathrm{Tnf}^{/-}$pups were indistinguishable from non-mutant controls with all animals surviving to adulthood with normalization of both body and spleen/body weight comparisons. Serum analysis of MWS Tnf/- pups showed attenuation of NLRP3 inflammsome related cytokines when compared to intact MWS pups. The skin of intact MWS pups exhibited strong neutrophilic and inflammatory macrophage infiltrations, which were normalized in $M W S T n f^{/-}$animals. Interestingly, $M W S \mathrm{Tnf}^{+/-}$pups also showed an intermediate protective effect on all of the aforementioned comparisons. To determine if TNF ablation could be recapitulated with therapeutic intervention, $M W S$ pups were treated with recombinant soluble TNF receptor (Etanercept 400ug/g sc EOD). Treatment provided similar phenotypic rescue and extended survival for an average of 22 days after cessation of treatment. Adult $M W S \mathrm{Tnf}^{-/}$mice at $>6$ months of age were found to have splenomegaly and elevated numbers of white blood cells when compared to non-mutant $\mathrm{Tnf}^{-{ }^{-} \text {lit- }}$ termates, implicating a role for other inflammatory mechanisms as mice age.

\section{Conclusion}

TNF plays an unexpected and significant role in murine inflammasomeopathies, which may have therapeutic implications for CAPS patients with incomplete responses to IL-1 targeted therapies.

\section{Authors' details}

${ }^{1}$ University of California San Diego, Pediatrics, La Jolla, USA. ${ }^{2}$ RWTH University Hospital Aachen, Internal Medicine III, Aachen, Germany.

Published: 28 September 2015

doi:10.1186/1546-0096-13-S1-O27

Cite this article as: McGeough et al:: A significant role for tumor necrosis factor in NIrp3 inflammasomeopathies. Pediatric Rheumatology 2015 13(Suppl 1):O27. 